\title{
The Singularities of Gravitational Fields of Static Thin Loop and Double Spheres Reveal the Impossibility of Singularity Black Holes
}

\author{
Xiaochun Mei \\ Institute of Innovative Physics in Fuzhou, Fuzhou, China \\ Email: ycwlyjs@yeah.net
}

Received April 21, 2013; revised May 22, 2013; accepted June 20, 2013

Copyright (C) 2013 Xiaochun Mei. This is an open access article distributed under the Creative Commons Attribution License, which permits unrestricted use, distribution, and reproduction in any medium, provided the original work is properly cited.

\begin{abstract}
In the classical Newtonian mechanics, the gravity fields of static thin loop and double spheres are two simple but foundational problems. However, in the Einstein's theory of gravity, they are not simple. In fact, we do not know their solutions up to now. Based on the coordinate transformations of the Kerr and the Kerr-Newman solutions of the Einstein's equation of gravity field with axial symmetry, the gravity fields of static thin loop and double spheres are obtained. The results indicate that, no matter how much the mass and density are, there are singularities at the central point of thin loop and the contact point of double spheres. What is more, the singularities are completely exposed in vacuum. Space near the surfaces of thin loop and spheres are highly curved, although the gravity fields are very weak. These results are inconsistent with practical experience and completely impossible. By reasonable analogy, black holes with singularity in cosmology and astrophysics are something illusive. Caused by the mathematical description of curved space-time, they do not exist in real world actually. If there are black holes in the universe, they can only be the types of the Newtonian black holes without singularities, rather than the Einstein's singularity black holes. In order to escape the puzzle of singularity thoroughly, the description of gravity should return to the traditional form of dynamics in flat space. The renormalization of gravity and the unified description of four basic interactions may be possible only based on the frame of flat space-time. Otherwise, theses problems can not be solved forever. Physicists should have a clear understanding about this problem.
\end{abstract}

Keywords: General Relativity; The Einstein's Equation of Gravity Field; Axially Symmetrical Solutions; Singularity; Kerr Metric; Kerr-Newman Metric; Gravitational Field of Static Thin Loop; Gravitational Field of Double Spheres Black Hole; Quasar; MECO

\section{Introduction}

According to the Einstein's theory of gravity, singularities exist at the centers of celestial bodies when material densities are great enough and gravity fields are strong enough. However, singularities are always confusing. Real world can not be infinite. If infinite appears in our theory, we have to argue whether the theory has something wrong. Making a general survey of scientific history, we see that physical progresses are often based on the elimination of infinite. Facing the problem of singularity in the Einstein's theory of gravity, such as singularity black holes, we should be skeptical rather than appreciative.

In fact, the author has proved that the present theory of singularity black hole is impossible by calculating the precise inner solutions of gravity field equations of hollow and solid spheres [1]. To avoid space curvature infinite at the center of solid sphere, we set an integral constant to be zero directly at present. However, according to the theory of differential equation, the integral constant should be deter-mined by the known boundary conditions of spherical surface, in stead of the metric at the spherical center. By considering that fact that the volumes of three dimensional hollow and solid spheres in curved space are different from those in flat space, the integral constants are proved to be nonzero. The results indicate that no matter what the masses and densities of hollow sphere and solid sphere are, there exist space-time singularities at the centers of hollow sphere and solid spheres.

Meanwhile, the intensity of pressure at the center point 
of solid sphere can not be infinite. That is to say, the material can not collapse towards the center of so-called black hole. At the center and its neighboring region of solid sphere, pressure intensities become negative values. There may be a region for hollow sphere in which pressure intensities may become negative values too. The common hollow and solid spheres in daily live can not have such impenetrable characteristics. The results only indicate that the singularity black holes predicated by general relativity are caused by the descriptive method of curved space-time actually. If black holes exist really in the universe, they can only be the Newtonian black holes, not the Einstein's black holes.

According to the practical observations by Rudolf E. Schild and Darryl J. Leiter [2], the centre of Quasar 0957 +561 which was considered to be a black hole is actually a close object, called a MECO (Massive Eternally Collapsing Object). Unlike an empty hole, it is surrounded by a strong magnetic field and material. This result challenged traditional astrophysics and cosmology. It implied that the current theory of singular black hole may be wrong. We have reason to ask such a question. Whether or not singularity black holes, predicted by general relativity, really exist in the universe?

In this paper, we discuss the gravitational fields of static thin loop and double spheres. Based on the coordinate transformations of the Kerr and the Kerr-Newman solutions of the Einstein's equation of gravity with axial symmetry, the gravitational fields of static thin loop and double spheres are calculated. The results indicate that, no matter what their masses and density are, the spatial curvatures at the central point of thin loop and the contact point of two spheres are infinite. What is more, the singularities are completely exposed in vacuum. The spaces nearby the surfaces of loop and spheres are highly curved, even though their masses are very small so that the gravitational fields are very weak.

These results are completely inconsistent with practical experience. They are very absurd and completely impossible. The only possible explanation is that the singularities are caused by the description method of curved space-time. By logical analogy, so-called singular black holes and white holes as well as wormholes which connect both holes in the current cosmology and astrophysics are something illusive. They have nothing to do with the real world actually. If there are black holes in nature, they can only be the type of the Newtonian black holes, i.e., in a certain region in which light can not escape but there is no singularity, rather than the Einstein's singularity black holes! In fact, as we know that our university is actually a great black hole by considering its mass and radius! However, we live in it normally. Where is singularity?
In order to escape the problem of singularity thoroughly, we should describe gravity in flat space-time. The author has proposed a scheme by transforming the geodesic equation of the Schwarzschild solution of the Einstein's equation of gravity field to flat space-time for description, the relativity revised Newtonian formula of gravity can be obtained [3]. The space-time singularity in the Einstein's theory of gravity becomes the original point $r=0$ in the Newtonian formula of gravity. It is proved that the formula can describe the procession of Mercury perihelion well.

When the formula is used to describe the universe expansion, the revised Friedmann equation can be obtained. Based on it, the high red-shift of Ia supernova can be explained well. We do not need the hypotheses of the accelerating universe and dark energy. It is also unnecessary for us to assume that non-baryon dark material is 5 - 6 times more than normal baryon material in the universe if they really exist. The some puzzle problems in cosmology such as the Hubble constant and the universal age can also be solved well.

\section{The Gravitational Field and Singularity of Static Thin Loop}

The gravitational field of static thin loop is discussed at first. As shown in Figure 1, a thin loop with mass $M$ and radius $b$ is placed on the $x-y$ plane. The center of ring is located at the origin point of spherical coordinate system. The ring is thin enough so that its cross section can be neglected comparing with its perimeter. It will be seen later that even though the cross section of loop is not zero, the result is also the same essentially. Because the mass distribution of thin loop has axial symmetry, the metric tensor of gravitational field does

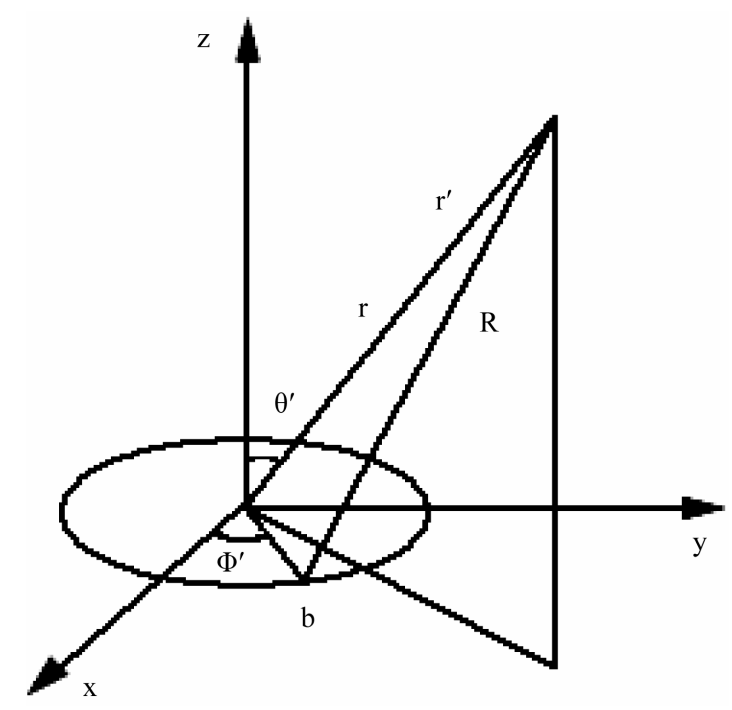

Figure 1. The gravity field of static thin loop. 
not depend on time $t$ and coordinate $\varphi$, so the four dimensional linear element can be written as

$$
\begin{aligned}
\mathrm{d} s^{2} & =g_{00}(r, \theta) \mathrm{d} t^{2}+g_{11}(r, \theta) \mathrm{d} r^{2} \\
& +g_{22}(r, \theta) r^{2} \mathrm{~d} \theta^{2} \\
& +g_{33}(r, \theta) r^{2} \sin ^{2} \theta \mathrm{d} \varphi^{2}
\end{aligned}
$$

By introducing coordinate transformations $t=t^{\prime}, \varphi \rightarrow \varphi^{\prime}, r \rightarrow r\left(r^{\prime}, \theta^{\prime}\right)$ and $\theta \rightarrow \theta\left(r^{\prime}, \theta^{\prime}\right)$, we can rewrite (1) as

$$
\begin{aligned}
\mathrm{d} s^{2} & =g_{00}^{\prime}\left(r^{\prime}, \theta^{\prime}\right) \mathrm{d} t^{\prime 2}+g_{11}^{\prime}\left(r^{\prime}, \theta^{\prime}\right) \mathrm{d} r^{\prime 2} \\
& +g_{22}^{\prime}\left(r^{\prime}, \theta^{\prime}\right) r^{\prime 2} \mathrm{~d} \theta^{\prime 2} \\
& +g_{33}^{\prime}\left(r^{\prime}, \theta^{\prime}\right) r^{\prime 2} \sin ^{2} \theta^{\prime} \mathrm{d} \varphi^{\prime 2} \\
& +g_{12}\left(r^{\prime}, \theta^{\prime}\right) r^{\prime} \mathrm{d} r^{\prime} \mathrm{d} \theta^{\prime}
\end{aligned}
$$

The Formulas (1) and (2) are with axial symmetry and can be used to describe the gravitational field of thin loop. Using these metrics in the Einstein's equation of gravity, we can obtain the concrete forms of metric tensor in principle. However, it is difficult to solve the equation of gravitational fields directly.

On the other hand, we know that there is a ready-made solution of gravitational field's equation with axial symmetry and two independent parameters, i.e., the Kerr solution [4]. If the solution of the Einstein's equation of gravity with the same symmetry and parameters is unique, we can obtain the solution of static mass distribution of thin loop by means of the coordinate transformations of the Kerr solution. Besides, we seem to have no other choice. The method is discussed below.

The Kerr solution with two free parameters is

$$
\begin{aligned}
\mathrm{d} s^{2}= & \left(1-\frac{2 \alpha r}{r^{2}+\beta^{2} \cos ^{2} \theta}\right) \mathrm{d} t^{2} \\
& -\frac{r^{2}+\beta^{2} \cos ^{2} \theta}{r^{2}+\beta^{2}-2 \alpha r} \mathrm{~d} r^{2}-\left(1+\frac{\beta^{2}}{r^{2}} \cos ^{2} \theta\right) r^{2} \mathrm{~d} \theta^{2} \\
& -\left[1+\frac{\beta^{2}}{r^{2}}+\frac{2 \alpha \beta^{2} \sin ^{2} \theta}{r\left(r^{2}+\beta^{2} \cos ^{2} \theta\right)}\right] r^{2} \sin ^{2} \mathrm{~d} \varphi^{2} \\
& +2 \frac{2 \alpha \beta \sin \theta}{r^{2}+\beta^{2} \cos ^{2} \theta} r \sin \theta \mathrm{d} t \mathrm{~d} \varphi
\end{aligned}
$$

At present, the Kerr metric is used to describe the gravity field of a rotating sphere, in which parameters $\alpha=G M, \beta=J / M,(c=1) . \quad \beta$ is considered to be the unit angle momentum. If we use (3) to describe the gravitational field of thin loop, $\alpha$ and $\beta$ will have different meanings. Because (3) contains a crossing item $\mathrm{d} t \mathrm{~d} \varphi$ which is related to time, the solution is dynamic one, rather than static. For static mass distribution, this item does not exist and should be canceled. We can remove it by the diagonalization of metric tensors. We have

$$
\begin{aligned}
& {\left[\begin{array}{ll}
g_{00} & g_{30} \\
g_{03} & g_{33}
\end{array}\right]} \\
& =\left[\begin{array}{cc}
1-\frac{2 \alpha r}{r^{2}+\beta^{2} \cos ^{2} \theta} & \frac{2 \alpha \beta \sin \theta}{r^{2}+\beta^{2} \cos ^{2} \theta} \\
\frac{2 \alpha \beta \sin \theta}{r^{2}+\beta^{2} \cos ^{2} \theta} & -1-\frac{\beta^{2}}{r^{2}}-\frac{2 \alpha \beta^{2} \sin ^{2} \theta}{r\left(r^{2}+\beta^{2} \cos ^{2} \theta\right)}
\end{array}\right]
\end{aligned}
$$

From the eigen equation

$$
\left|\begin{array}{cc}
g_{00}-\lambda & g_{03} \\
g_{30} & g_{33}-\lambda
\end{array}\right|=0
$$

we get

$$
\begin{aligned}
\lambda_{1}= & \frac{1}{2}\left[g_{00}+g_{33}+\sqrt{\left(g_{00}-g_{33}\right)^{2}+4 g_{03} g_{30}}\right] \\
= & \frac{1}{2}\left[\left(2-\frac{2 \alpha r}{r^{2}+\beta^{2} \cos ^{2} \theta}+\frac{\beta^{2}}{r^{2}}+\frac{2 \alpha \beta^{2} \sin ^{2} \theta}{r\left(r^{2}+\beta^{2} \cos ^{2} \theta\right)}\right)^{2}+\frac{16 \alpha^{2} \beta^{2} \sin ^{2} \theta}{\left(r^{2}+\beta^{2} \cos ^{2} \theta\right)^{2}}\right]^{\frac{1}{2}} \\
& -\frac{1}{2}\left[\frac{2 \alpha r}{r^{2}+\beta \cos ^{2} \theta}+\frac{\alpha^{2}}{r^{2}}+\frac{2 \alpha \beta^{2} \sin ^{2} \theta}{r\left(r^{2}+\beta^{2} \cos ^{2} \theta\right)}\right] \\
= & \frac{1}{2}\left[g_{00}+g_{33}-\sqrt{\left(g_{00}-g_{33}\right)^{2}+4 g_{03} g_{30}}\right] \\
= & -\frac{1}{2}\left[\left(2-\frac{2 \alpha r}{r^{2}+\beta^{2} \cos ^{2} \theta}+\frac{\beta^{2}}{r^{2}}+\frac{2 \alpha \beta^{2} \sin ^{2} \theta}{r\left(r^{2}+\beta^{2} \cos ^{2} \theta\right)}\right)^{2}+\frac{16 \alpha^{2} \beta^{2} \sin ^{2} \theta}{\left(r^{2}+\beta^{2} \cos ^{2} \theta\right)^{2}}\right]^{\frac{1}{2}} \\
& +\frac{1}{2}\left[\frac{2 \alpha r}{r^{2}+\beta \cos ^{2} \theta}+\frac{\alpha^{2}}{r^{2}}+\frac{2 \alpha \beta^{2} \sin ^{2} \theta}{r\left(r^{2}+\beta^{2} \cos ^{2} \theta\right)}\right]
\end{aligned}
$$


The orthogonal transformations of coordinates are

$$
\left[\begin{array}{c}
\mathrm{d} t \\
r \sin \theta \mathrm{d} \varphi
\end{array}\right]=\left[\begin{array}{ll}
\frac{g_{30}}{\sqrt{g_{03}^{2}+\left(g_{00}-\lambda_{1}\right)^{2}}} & \frac{g_{30}}{\sqrt{g_{03}^{2}+\left(g_{00}-\lambda_{2}\right)^{2}}} \\
\frac{g_{00}-\lambda_{1}}{\sqrt{g_{03}^{2}+\left(g_{00}-\lambda_{1}\right)^{2}}} & \frac{g_{00}-\lambda_{2}}{\sqrt{g_{03}^{2}+\left(g_{00}-\lambda_{2}\right)^{2}}}
\end{array}\right] \times\left[\begin{array}{c}
\mathrm{d} t^{\prime} \\
r \sin \theta \mathrm{d} \varphi^{\prime}
\end{array}\right]
$$

The inverse transformation is

$$
\left[\begin{array}{c}
\mathrm{d} t^{\prime} \\
r \sin \theta \mathrm{d} \varphi^{\prime}
\end{array}\right]=\left[\begin{array}{cc}
\frac{\left(g_{00}-\lambda_{1}\right) \sqrt{g_{03}^{2}+\left(g_{00}-\lambda_{1}\right)^{2}}}{g_{03}\left(\lambda_{1}-\lambda_{2}\right)} & \frac{-\sqrt{g_{03}^{2}+\left(g_{00}-\lambda_{1}\right)^{2}}}{\left(\lambda_{1}-\lambda_{2}\right)} \\
\frac{-\left(g_{00}-\lambda_{2}\right) \sqrt{g_{03}^{2}+\left(g_{00}-\lambda_{1}\right)^{2}}}{g_{03}\left(\lambda_{1}-\lambda_{2}\right)} & \frac{\sqrt{g_{03}^{2}+\left(g_{00}-\lambda_{2}\right)^{2}}}{\left(\lambda_{1}-\lambda_{2}\right)}
\end{array}\right] \times\left[\begin{array}{c}
\mathrm{d} t \\
r \sin \theta \mathrm{d} \varphi
\end{array}\right]
$$

By the transformation, we can cancel crossing item containing $\mathrm{d} t \mathrm{~d} \varphi$ and get

$$
g_{00} \mathrm{~d} t^{2}+g_{33} r^{2} \sin ^{2} \theta \mathrm{d} \varphi^{2}+2 g_{03} r \sin \theta \mathrm{d} t \mathrm{~d} \varphi=\lambda_{1} \mathrm{~d} t^{2}+\lambda_{2} r^{2} \sin ^{2} \theta \mathrm{d} \varphi^{\prime 2}
$$

Substitute (9) into (3), we can transform it into the diagonal form. For the consistency of notations, we set $t^{\prime} \rightarrow t, \varphi^{\prime} \rightarrow \varphi$ again and obtain the result

$$
\begin{aligned}
& \mathrm{d} s^{2}=\frac{1}{2} \\
& \left.\qquad\left\{\left[2-\frac{2 \alpha r}{r^{2}+\beta^{2} \cos ^{2} \theta}+\frac{\beta^{2}}{r^{2}}+\frac{2 \alpha \beta^{2} \sin ^{2} \theta}{r\left(r^{2}+\beta^{2} \cos ^{2} \theta\right)}\right)^{2}+\frac{16 \alpha^{2} \beta^{2} \sin ^{2} \theta}{\left(r^{2}+\beta^{2} \cos ^{2} \theta\right)^{2}}\right]^{\frac{1}{2}}-\frac{2 \alpha r}{r^{2}+\beta^{2} \cos ^{2} \theta}-\frac{\beta^{2}}{r^{2}}-\frac{2 \alpha \beta^{2} \sin ^{2} \theta}{r\left(r^{2}+\beta^{2} \cos ^{2} \theta\right)}\right\} \mathrm{d} t^{2} \\
& -\frac{r^{2}+\beta^{2} \cos ^{2} \theta}{r^{2}+\beta^{2}-2 \alpha r} \mathrm{~d} r^{2}-\left(1+\frac{\beta^{2}}{r^{2}} \cos ^{2} \theta\right) r^{2} \mathrm{~d} \theta^{2} \\
& -\frac{1}{2}\left\{\left[\left(2-\frac{2 \alpha r}{r^{2}+\beta^{2} \cos ^{2} \theta}+\frac{\beta^{2}}{r^{2}}+\frac{2 \alpha \beta^{2} \sin ^{2} \theta}{r\left(r^{2}+\beta^{2} \cos ^{2} \theta\right)}\right)^{2}+\frac{16 \alpha^{2} \beta^{2} \sin ^{2} \theta}{\left(r^{2}+\beta^{2} \cos ^{2} \theta\right)}\right]^{2}+\frac{2 \alpha r}{r^{2}+\beta^{2} \cos ^{2} \theta}+\frac{\beta^{2}}{r^{2}}+\frac{2 \alpha \beta^{2} \sin ^{2} \theta}{r\left(r^{2}+\beta^{2} \cos ^{2} \theta\right)}\right\} \\
& \times r^{2} \sin ^{2} \theta \mathrm{d} \varphi^{2}
\end{aligned}
$$

The Formula (10) has the form of (1), so we can use it to describe the gravitational field of static thin loop.

On the other hand, we know in general relativity that only by comparing with the Newtonian theory in the weak field when $r$ is great enough, the integral constant of the solution of the Einstein's equation of gravity field can be determined. According to this principle, we have relation

$$
g_{00}=1+2 \psi
$$

Here $\psi$ is the Newtonian gravity potential. Now let's discuss the concrete form of $\psi$ for a thin loop. As is shown in Figure 1, suppose that the coordinates of observation points are

$x_{0}=r^{\prime} \sin \theta^{\prime} \cos \varphi^{\prime}, y_{0}=r^{\prime} \sin \theta^{\prime} \sin \varphi^{\prime}$ and $z_{0}=r^{\prime} \cos \theta^{\prime}$. The coordinates of a point on the surface of thin loop are $x=b \cos \varphi, y=b \sin \varphi, z=0$. The distance between these two points is

$$
\begin{aligned}
R & =\sqrt{\left(x_{0}-x\right)^{2}+\left(y_{0}-y\right)^{2}+\left(z_{0}-z\right)^{2}} \\
& =\sqrt{r^{\prime 2}+b^{2}-2 r^{\prime} b \sin \theta^{\prime} \cos \left(\varphi-\varphi^{\prime}\right)}
\end{aligned}
$$

For symmetry and simplicity, we take $\varphi^{\prime}=0$, so the Newtonian potential of thin loop is

$$
\psi=-\int \frac{G \mathrm{~d} M}{R}=-\int_{0}^{\pi} \frac{2 G \rho b \mathrm{~d} \varphi}{\sqrt{r^{\prime 2}+b^{2}-2 r^{\prime} b \sin \theta^{\prime} \cos \varphi}}
$$

Here $M, \rho$ and $b$ are mass, linear density and radius of thin loop individually. Let $\varphi=\pi-\phi^{\prime}, \mathrm{d} \varphi=-\mathrm{d} \phi^{\prime}$, $-\cos \varphi=\cos \phi^{\prime}=1-2 \sin ^{2}\left(\phi^{\prime} / 2\right)$, and put them into (13), we get 


$$
\psi=\int_{\pi}^{0} \frac{2 G \rho b \mathrm{~d} \varphi^{\prime}}{\sqrt{r^{\prime 2}+b^{2}+2 r^{\prime} b \sin \theta-4 r^{\prime} b \sin \theta^{\prime} \sin ^{2}\left(\phi^{\prime} / 2\right)}}
$$

Then let $\phi^{\prime \prime}=\phi^{\prime} / 2$ again, (14) can be written as

$$
\begin{aligned}
\psi & =-\int_{0}^{\pi / 2} \frac{4 G \rho b \mathrm{~d} \varphi^{\prime \prime}}{\sqrt{r^{\prime 2}+b^{2}+2 r^{\prime} b \sin \theta^{\prime}-4 r^{\prime} b \sin \theta^{\prime} \sin ^{2} \varphi^{\prime \prime}}} \\
& =-\frac{4 G \rho b}{\sqrt{r^{\prime 2}+b^{2}+2 r^{\prime} b \sin \theta^{\prime}}} \int_{0}^{\pi / 2} \frac{\mathrm{d} \phi^{\prime \prime}}{\sqrt{1-k^{2} \sin ^{2} \phi^{\prime \prime}}}
\end{aligned}
$$

We have $k^{2}=4 r^{\prime} b \sin \theta^{\prime} /\left(r^{\prime 2}+b^{2}+2 r^{\prime} b \sin \theta^{\prime}\right)$ in the formula. Let

$$
K\left(k^{2}\right)=\int_{0}^{\pi / 2} \frac{\mathrm{d} \varphi^{\prime \prime}}{\sqrt{1-k^{2} \sin ^{2} \varphi^{\prime \prime}}}
$$

(16) is just the first kind ellipse function. When $r^{\prime} \rightarrow \infty$, we have

$$
\begin{aligned}
& \frac{1}{\sqrt{r^{\prime 2}+b^{2}+2 r^{\prime} b \sin \theta^{\prime}}} \\
& =\frac{1}{r^{\prime}}\left[1-\frac{b \sin \theta^{\prime}}{r^{\prime}}+\frac{b^{2}\left(3 \sin ^{2} \theta^{\prime}-1\right)}{2 r^{\prime 2}}+\cdots\right]
\end{aligned}
$$

Substituting (16) and (18) into (15) and considering $2 \pi \rho b=M$, we get

$$
\psi=-\frac{G M}{r^{\prime}}\left[1-\frac{b^{2}\left(2-11 \sin ^{2} \theta^{\prime}\right)}{4 r^{\prime 2}}+\cdots\right]
$$

On the other hand, we can expand $g_{\mu v}$ into the power series about $1 / r$ and write (10) as

$$
\begin{aligned}
& \mathrm{d} s^{2} \\
& =\left(1-\frac{2 \alpha}{r}+\frac{2 \alpha \beta^{2} \cos ^{2} \theta}{r^{3}}+\cdots\right) \mathrm{d} t^{2} \\
& -\left(1+\frac{2 \alpha}{r}-\frac{2 \alpha+\beta^{2} \sin ^{2} \theta}{r^{2}}+\frac{12 \alpha \beta^{2}-48 \alpha^{3}}{r^{3}}+\cdots\right) \mathrm{d} r^{2} \\
& -\left(1+\frac{\beta^{2}}{r^{2}} \cos ^{2} \theta\right) r^{2} \mathrm{~d} \theta^{2} \\
& -\left(1+\frac{\beta^{2}}{r^{2}}+\frac{2 \alpha \beta^{2} \sin ^{2} \theta}{r^{3}}+\cdots\right) r^{2} \sin ^{2} \theta \mathrm{d} \varphi^{2}
\end{aligned}
$$

By comparing (11) and the item $g_{00}$ in (20) up to the order $r^{-1}$, we get

$$
1-\frac{2 \alpha}{r}=1-\frac{2 G M}{r^{\prime}}
$$

Let constant $\alpha=G M$, we get $r=r^{\prime}$. However, the relation is only suitable for the situation when the mass of thin loop is concentrated at the center point of the loop.
In order to obtain the more accurate gravity potential of thin loop, we should consider higher order items. There are no items containing $r^{-2}$ order in (19). By considering the items containing order up to $r^{-3}$, we have

$$
\begin{aligned}
& 1-\frac{2 G M}{r}+\frac{2 G M \beta^{2} \cos ^{2} \theta}{r^{3}} \\
& =1-\frac{2 G M}{r^{\prime}}+\frac{2 G M b^{2}\left(0.5-2.75 \sin ^{2} \theta^{\prime}\right)}{r^{\prime 3}}
\end{aligned}
$$

We see that the function forms on the two sides of (22) are different. It means that the solution of the Einstein's equation of gravity can not asymptotically coincide with the Newtonian theory of gravity automatically in this case. In order to make them asymptotically consistent, further transformation is needed. Because constant $\beta$ has the dimension of length, we can take $\beta=b$. Because we always have $\cos ^{2} \theta \geq 0$ but may have $0.5-2.75 \sin ^{2} \theta^{\prime}<0$, so we have $0.5-2.75 \sin ^{2} \theta^{\prime} \neq \cos ^{2} \theta$ in general. Therefore, we have $r \neq r^{\prime}$ in (22). However, we can set $\theta=\theta^{\prime}$ so that (22) becomes

$$
\frac{1}{r}-\frac{b^{2} \cos ^{2} \theta^{\prime}}{r^{3}}=\frac{1}{r^{\prime}}-\frac{b^{2}\left(0.5-2.75 \sin ^{2} \theta^{\prime}\right)}{r^{\prime 3}}
$$

Let $A=b^{2} \cos ^{2} \theta^{\prime}, B=b^{2}\left(0.5-2.75 \sin ^{2} \theta^{\prime}\right)$ and by considering the condition $r^{\prime} \gg b$, the only real number solution of (23) is

$$
\begin{aligned}
\frac{1}{r} & =\left[\frac{1}{2 A r^{\prime 3}}\left(B-r^{\prime 2}\right)+\frac{\mathrm{i}}{2 A^{3 / 2} r^{\prime 3}} \sqrt{4 r^{\prime 6}-A\left(B-r^{\prime 2}\right)^{2}}\right]^{\frac{1}{3}} \\
& +\left[\frac{1}{2 A r^{\prime 3}}\left(B-r^{\prime 2}\right)-\frac{\mathrm{i}}{2 A^{3 / 2} r^{\prime 3}} \sqrt{4 r^{\prime 6}-A\left(B-r^{\prime 2}\right)^{2}}\right]^{\frac{1}{3}} \\
& =(a+\mathrm{i} b)^{\frac{1}{3}}+(a-\mathrm{i} b)^{\frac{1}{3}}=2 Q \cos (\delta / 3)
\end{aligned}
$$

Here

$$
\begin{aligned}
& a=\frac{B-r^{\prime 2}}{2 A r^{\prime}}, b=\frac{\sqrt{4 r^{\prime 6}-A\left(B-r^{\prime 2}\right)^{2}}}{2 A^{3 / 2} r^{\prime 3}} \\
& Q=\left(a^{2}+b^{2}\right)^{1 / 6}, \operatorname{tg} \frac{b}{a}=\delta .
\end{aligned}
$$

So we can write $r=r\left(r^{\prime}, \theta^{\prime}\right)$ and obtain

$$
\begin{aligned}
\mathrm{d} r & =\frac{\mathrm{d} r}{\mathrm{~d} r^{\prime}} \mathrm{d} r^{\prime}+\frac{\mathrm{d} r}{\mathrm{~d} \theta^{\prime}} \mathrm{d} \theta^{\prime} \\
& =T\left(r^{\prime}, \theta^{\prime}\right) \mathrm{d} r^{\prime}+V\left(r^{\prime}, \theta^{\prime}\right) \mathrm{d} \theta^{\prime}
\end{aligned}
$$

The concrete forms of functions $T\left(r^{\prime}, \theta^{\prime}\right)$ and $V\left(r^{\prime}, \theta^{\prime}\right)$ are unimportant, so we do not write them out here. Now we substitute (26) into (10) and obtain the metric of gravitational equation of thin loop which has the form of (2) with $r=r\left(r^{\prime}, \theta^{\prime}\right)$ 


$$
\begin{aligned}
& \mathrm{d} s^{2} \\
& =\frac{1}{2}\left\{\left[\left(2-\frac{2 \alpha r}{r^{2}+b^{2} \cos ^{2} \theta^{\prime}}+\frac{b^{2}}{r^{2}}+\frac{2 \alpha b^{2} \sin ^{2} \theta^{\prime}}{r\left(r^{2}+b^{2} \cos ^{2} \theta^{\prime}\right)}\right)^{2}+\frac{16 \alpha^{2} b^{2} \sin ^{2} \theta^{\prime}}{\left(r^{2}+b^{2} \cos ^{2} \theta^{\prime}\right)^{2}}\right]^{\frac{1}{2}}-\frac{2 \alpha r}{r^{2}+b^{2} \cos ^{2} \theta}-\frac{b^{2}}{r^{2}}-\frac{2 \alpha b^{2} \sin ^{2} \theta^{\prime}}{r\left(r^{2}+b^{2} \cos ^{2} \theta^{\prime}\right)}\right\} \mathrm{d} t^{\prime 2} \\
& -\frac{r^{2}+b^{2} \cos ^{2} \theta^{\prime}}{r^{2}+b^{2}-2 \alpha r} T^{2}\left(r^{\prime}, \theta^{\prime}\right) \mathrm{d} r^{\prime 2}-2 \frac{r^{2}+b^{2} \cos ^{2} \theta^{\prime}}{r^{2}+b^{2}-2 \alpha r} T\left(r^{\prime}, \theta^{\prime}\right) V\left(r^{\prime}, \theta^{\prime}\right) \mathrm{d} r^{\prime} \mathrm{d} \theta^{\prime} \\
& -\left[\left(1+\frac{b^{2}}{r^{2}} \cos ^{2} \theta^{\prime}\right) \frac{r^{2}}{r^{\prime 2}}+\frac{r^{2}+b \cos ^{2} \theta^{\prime}}{r^{2}+b^{2}-2 \alpha b} \frac{\left.V^{\prime}, \theta^{\prime}\right)}{r^{\prime 2}}\right] \\
& \times r^{\prime 2} \mathrm{~d} \theta^{\prime 2}-\frac{r^{2}}{2 r^{\prime 2}} \\
& \cdot\left\{\left[\left(2-\frac{2 \alpha r}{r^{2}+b^{2} \cos ^{2} \theta^{\prime}}+\frac{b^{2}}{r^{2}}+\frac{2 a b^{2} r \sin ^{2} \theta^{\prime}}{r\left(r^{2}+b^{2} \cos ^{2} \theta^{\prime}\right)^{2}}\right)^{2}+\frac{16 \alpha^{2} b^{2} \sin ^{2} \theta^{\prime}}{\left(r^{2}+b^{2} \cos ^{2} \theta^{\prime}\right)^{2}}\right]^{\frac{1}{2}}+\frac{2 \alpha r}{r^{2}+b^{2} \cos ^{2} \theta^{\prime}}+\frac{b^{2}}{r^{2}}+\frac{2 \alpha b^{2} \sin ^{2} \theta^{\prime}}{r\left(r^{2}+b^{2} \cos ^{2} \theta^{\prime}\right)}\right\} \\
& \times r^{\prime 2} \sin ^{2} \theta^{\prime} \mathrm{d} \varphi^{\prime 2}
\end{aligned}
$$

As is shown in Figure 1, or by the definition of coordinate systems, we have both $r^{\prime}=0$ and $r=0$ simultaneously for the original points of two coordinate systems. When $r^{\prime}=0$, we have $r=0$ in (27) which leads to $g_{00} \rightarrow \infty, g_{22} \rightarrow \infty$, and $g_{33} \rightarrow \infty$. The result shows that a singularity will appear at the centre of thin loop. This singularity is completely exposed in vacuum, no matter how much the mass or density of thin loop is, even they are very small. The singularity is essential one which can not be removed by coordinate transformation. This result is absurd and unacceptable, for it obviously violates common experience. It does not like the singularity of the Schwarzschild solution which is considered to hide in the center of huge mass and unobservable directly so that physicists can tolerate its existence.

Besides, it can be proved that the space nearby the surface of thin loop is also high curved. Because of $\alpha \ll b$, we can let $\alpha \rightarrow 0$ for approximation. In the nearby region of thin loop's surface, we take $\theta^{\prime} \approx \pi / 2$, so (27) becomes

$$
\begin{aligned}
\mathrm{d} s^{2} & =\mathrm{d} t^{\prime 2}-\frac{r^{2}}{r^{2}+b^{2}} T^{2}\left(r^{\prime}, \pi / 2\right) \mathrm{d} r^{\prime 2} \\
& -\left[\frac{r^{2}}{r^{\prime 2}}+\frac{r^{2} V^{2}\left(r^{\prime}, \pi / 2\right)}{r^{\prime 2}\left(r^{2}+b^{2}\right)}\right] r^{\prime 2} \mathrm{~d} \theta^{\prime 2} \\
& -\left(\frac{r^{2}}{r^{\prime 2}}+\frac{b^{2}}{r^{\prime 2}}\right) r^{\prime 2} \mathrm{~d} \varphi^{\prime 2} \\
& -\frac{2 r^{2}}{r^{\prime}\left(r^{2}+b^{2}\right)} T\left(r^{\prime}, \pi / 2\right) V\left(r^{\prime}, \pi / 2\right) r^{\prime} \mathrm{d} r^{\prime} \mathrm{d} \theta^{\prime}
\end{aligned}
$$

Take $b=0.67,(23)$ becomes

$$
\frac{1}{r}=\frac{1}{r^{\prime}}+\frac{1}{r^{\prime 3}}
$$

or

$$
r=\frac{r^{\prime 3}}{r^{\prime 2}+1}
$$

By considering Equation (26), we have

$$
T\left(r^{\prime}, \pi / 2\right)=\frac{r^{\prime 4}+3 r^{\prime 2}}{\left(r^{\prime 2}+1\right)^{2}}, V\left(r^{\prime}, \pi / 2\right)=0
$$

Take $r^{\prime}=b=0.67$, we have $r=0.21$. Using these values in (28), we obtain $g_{11}^{\prime}=-0.10, g_{22}^{\prime}=-0.10$ and $g_{33}^{\prime}=-1.10$. So the space nearby the surface of thin loop is highly curved. The result does not agree with practical experiences completely. On the surface of thin loop, the gravity is very weak and space should be nearly flat with $g_{11}^{\prime}=g_{22}^{\prime}=g_{33}^{\prime} \approx-1$.

Because the curvature of space is a quantity which can be measured directly, the solution (27) is improper for the gravitational field of thin loop. In fact, according to the result (13) of the Newtonian theory, at the center point $r^{\prime}=0$, the gravitational potential of loop is a limited constant with

$$
\psi=-\int_{0}^{\pi} 2 G \rho \mathrm{d} \varphi=-2 \pi G \rho=-\frac{G M}{b}
$$

Because $\psi$ is a constant, the gravity at the center point of loop is zero. This agrees with practical experiences. The essential problem is that for such simple and foundational material distribution, if (27) is improper, what is the correct solution for the Einstein's equation of 
gravity? Can we find another solution? If can, how can we deal with the problem of the uniqueness of theory?

On the other hand, let $r^{2}+b^{2}-2 \alpha r=0$ in (27), we have $r=\alpha \pm \sqrt{\alpha^{2}-b^{2}}$. By taking $M \sim 1 \mathrm{Kg}$ and $b=1 \mathrm{~m}$, we have $\alpha=G M / c^{2}=7.41 \times 10^{-28}$ and $\alpha \ll b$. So if we take $r=\alpha \pm \sqrt{\alpha^{2}-b^{2}}, r$ would not be a real number. Therefore, $r^{\prime}$ would not be a real number too.

The second singularity of (27) determined by relation $r=\alpha \pm \sqrt{\alpha^{2}-b^{2}}$ does not exist. In the Kerr solution, $r=\alpha \pm \sqrt{\alpha^{2}-b^{2}}$ describers a surface of elliptical sphere which represents the event horizon of black hole. But for the gravitational field of thin loop, because equation $r^{2}+b^{2}-2 \alpha r=0$ has no real solution in general situa- tions, the event horizon does not exist.

Next, we discuss the situation when the cross section of thin loop is not zero. In this case, the gravitation field has three independent parameters. The third is the radium of loop's cross section. As we have known that the KerrNewman metric is one with axial symmetry and three independent parameters [5]. At present, it is used to describe the external gravitational field of revolving charged sphere. If the solution of the Einstein's equation of gravitational field with three parameters and axial symmetry is unique, by the coordinate transformation, we can also reach the gravitational field of loop with cross section based on the Kerr-Newman metric. By the same method of metric tensor's diagonalization, we can write the Kerr-Newman metric as

$$
\begin{aligned}
& \mathrm{d} s^{2} \\
& =\frac{1}{2}\left\{\left[\left(2-\frac{2 \alpha r-Q^{2}}{r^{2}+\beta^{2} \cos ^{2} \theta}+\frac{\beta^{2}}{r^{2}}+\frac{2 \alpha \beta^{2} \sin ^{2} \theta}{r\left(r^{2}+\beta^{2} \cos ^{2} \theta\right)}\right)^{2}+\frac{16 \alpha^{2} \beta^{2} \sin ^{2} \theta}{\left(r^{2}+\beta^{2} \cos ^{2} \theta\right)^{2}}\right]^{\frac{1}{2}}-\frac{2 \alpha r-Q^{2}}{r^{2}+\beta^{2} \cos ^{2} \theta}-\frac{\beta^{2}}{r^{2}}-\frac{2 \alpha \beta^{2} \sin ^{2} \theta}{r\left(r^{2}+\beta^{2} \cos ^{2} \theta\right)}\right\} \mathrm{d} t^{2} \\
& -\frac{r^{2}+\beta^{2} \cos ^{2} \theta}{r^{2}+\beta^{2}-2 \alpha r-Q^{2}} \mathrm{~d} r^{2}-\left(1+\frac{\beta^{2}}{r^{2}} \cos ^{2} \theta\right) r^{2} \mathrm{~d} \theta^{2} \\
& -\frac{1}{2}\left\{\left[\left(2-\frac{2 \alpha r-Q^{2}}{r^{2}+\beta^{2} \cos ^{2} \theta}+\frac{\beta^{2}}{r^{2}}+\frac{2 \alpha \beta^{2} \sin ^{2} \theta}{r\left(r^{2}+\beta^{2} \cos ^{2} \theta\right)}\right)^{2}+\frac{16 \alpha^{2} \beta^{2} \sin ^{2} \theta}{\left(r^{2}+\beta^{2} \cos ^{2} \theta\right)^{2}}\right]^{2}+\frac{2 \alpha r-Q^{2}}{r^{2}+\beta^{2} \cos ^{2} \theta}+\frac{\beta^{2}}{r^{2}}+\frac{2 \alpha \beta^{2} \sin ^{2} \theta}{r\left(r^{2}+\beta^{2} \cos ^{2} \theta\right)}\right\} \\
& \quad \cdot r^{2} \sin ^{2} \theta \mathrm{d} \varphi^{2}
\end{aligned}
$$

According to (32), when $r \gg \alpha$ and $r \gg \beta$, we have

$$
g_{00}=1-\frac{2 \alpha}{r}+\frac{Q^{2}}{r^{2}}+\frac{2 \alpha \beta^{2} \cos ^{2} \theta^{2}}{r^{3}}+\cdots
$$

On the other hand, when the area of thin loop's cross section is considered, the Newtonian potential of gravity field is very complex. We do not discuss it in detail but can get the same conclusion by the simple estimation. Suppose that the radius of thin loop's cross section is $h$, when $r \gg b, r \gg h$ and $h \sim b$, due to the axial symmetry, we can always write the Newtonian gravity potential as

$$
\psi=-\frac{G M}{r^{\prime}}+\frac{f_{1}\left(\theta^{\prime}, b, h\right)}{r^{\prime 2}}+\frac{f_{2}\left(\theta^{\prime}, b, h\right)}{r^{\prime 3}}+\cdots
$$

Similar to the discussion above, when $r \rightarrow \infty$, by considering terms up to order $r^{-2}$, we obtain from (11), (33) and (34)

$$
-\frac{G M}{r}+\frac{Q^{2}}{2 r^{2}}=-\frac{G M}{r^{\prime}}+\frac{f_{1}\left(\theta^{\prime}, b, h\right)}{r^{\prime 2}}
$$

Let $x=1 / r$ and $x^{\prime}=1 / r^{\prime}$, we can get from (35)

$$
x=\frac{G M+\sqrt{(G M)^{2}+2 Q^{2}\left(f_{1} x^{\prime 2}-G M x^{\prime}\right)}}{Q^{2}}
$$

When $x^{\prime} \rightarrow \infty$, we have also $x \rightarrow \infty$. That is to say, when $r^{\prime}=0$, we have $r=0$. Substitute (36) into (32), we can get the metric of loop with cross section. The singularity still exists at the center point of loop which is 
also exposed in vacuum. Space nearby the surface of loop is also highly curved. The situation is completely the same as that when the area of cross section of thin loop is neglected.

\section{The Gravitational Field and Singularity of Static Double Spheres}

As shown in Figure 2, the masses and radius of double spheres are $M$ and $b$. The centers of two spheres are located at the points $\pm b$ on the $z$ axis individually. It is obvious that the gravity field also has axial symmetry and two parameters and can be obtained through the coordinate transformation of the Kerr solution. For this problem, the Newtonian potential is

$$
\begin{aligned}
& \psi \\
& =-G M\left(\frac{1}{r_{1}}+\frac{1}{r_{2}}\right) \\
& =-G M\left(\frac{1}{\sqrt{r^{\prime 2}+b^{2}+2 b r^{\prime} \cos \theta}}+\frac{1}{\sqrt{r^{\prime 2}+b^{2}-2 b r^{\prime} \cos \theta}}\right)
\end{aligned}
$$

Here $r_{1}$ and $r_{2}$ are the distances between the center of two spheres and the certain point in space, $r^{\prime}$ is the original point of coordinate system and $\theta$ is the angle between $r^{\prime}$ and $z$ axis. When $r^{\prime} \gg b$, we have

$$
\psi=-\frac{2 G M}{r^{\prime}}\left(1-\frac{b^{2}-3 b^{2} \cos ^{2} \theta}{2 r^{\prime 2}}\right)
$$

From (11), (20) and (38) we get relationship

$$
\begin{aligned}
& 1-\frac{2 \alpha}{r}+\frac{2 \alpha \beta^{2} \cos ^{2} \theta}{r^{3}} \\
& =1-\frac{4 G M}{r^{\prime}}+\frac{2 G M b^{2}\left(1-3 \cos ^{2} \theta^{\prime}\right)}{r^{\prime 3}}
\end{aligned}
$$

Take $\alpha=2 G M, \beta=b, \theta=\theta^{\prime}$, we have

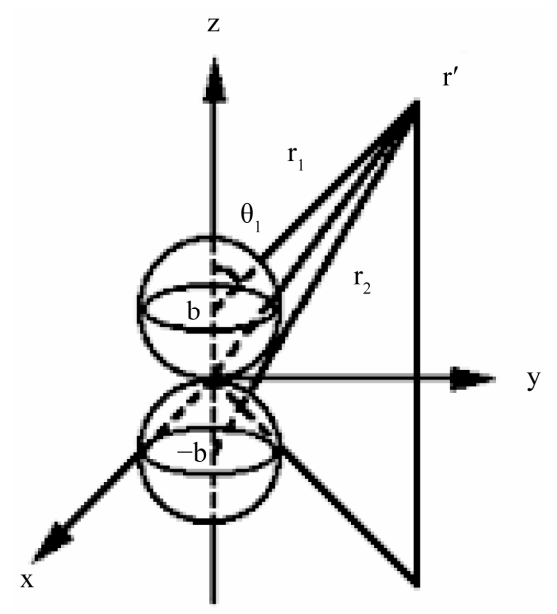

Figure 2. The gravity field of static double spheres.

$$
\frac{1}{r}-\frac{b^{2} \cos ^{2} \theta^{\prime}}{r^{3}}=\frac{1}{r^{\prime}}-\frac{b^{2}\left(1-3 \cos ^{2} \theta^{\prime}\right)}{2 r^{\prime 3}}
$$

Let $A=b^{2} \cos ^{2} \theta^{\prime}, B=b^{2}\left(1-3 \cos ^{2} \theta^{\prime}\right) / 2$ in (40), we can obtain the relations similar to (24) and (26). By considering (10), the metrics of static double spheres can be obtained. It also agrees with the form of (27). Further more, it is the same that we have $r=0$ and $r^{\prime}=0$ simultaneously. So there is a singularity at the contact point of double spheres with $g_{00} \rightarrow \infty, g_{22} \rightarrow \infty$, $g_{33} \rightarrow \infty$ and $g_{23} \rightarrow \infty$. Take $b=\sqrt{2}$ and $\theta^{\prime}=\pi / 2$, (40) becomes

$$
\frac{1}{r}=\frac{1}{r^{\prime}}-\frac{1}{r^{\prime 3}} \quad \text { or } \quad r=\frac{r^{\prime 3}}{r^{\prime 2}-1}
$$

Take $M=1 \mathrm{Kg}$, i.e., the gravitational field is very weak so that we can let $\alpha \rightarrow 0$ in (26) and get the formula similar to (30) with

$$
T\left(r^{\prime}, \theta^{\prime}\right)=\frac{r^{\prime 4}-3 r^{\prime 2}}{\left(r^{\prime 2}-1\right)^{2}}, V\left(r^{\prime}, \theta^{\prime}\right)=0
$$

Take $r^{\prime}=2$, we get $r=2.67$ and $T=0.44$. Substitute the values in (28), we obtain $g_{11}=-0.15$,

$g_{22}=-1.78$ and $g_{33}=-2.28$. It means that the space nearby the surfaces of two spheres is also high curved. However, this is impossible. In the weak field, we should have $g_{11}=g_{22}=g_{33} \approx-1$. More serious is that when $r^{\prime}<1, \quad r$ becomes a negative number according to (41) so that it is meaningless. So (26) is also unsuitable for the gravitational field of static double spheres.

In fact, there are many other axial symmetry distributions of masses with two or three parameters. For example, three spheres which are superposed one by one along a straight line, two cones which are superposed with their cusps meeting together, as well as the hollow column and so on. In principle, all of their gravitational fields can be obtained by means of the coordinate transformations based on the Kerr solution and the Kerr-Newman solutions. This method is unique actually. However, we can imagine that same problem will occur in all cases. The singularities would exist at some points and were exposed in vacuum. The spaces nearby the surfaces of objects are highly curved under the conditions of weak fields. All of them can not coincide with practical experiences.

\section{Conclusions}

According to the singularity theorem proved by Stephen Hawking, space-time singularities existed commonly and unavoidably in the Einstein's theory of gravity [6]. It is now believed that black holes are created through the collapse of material. Because black holes are considered to be hidden at the centers of super-massive mass with 
very high density, for example, at the centers of quasars and galaxies so that they can not be observed directly, physicists can tolerate their existence at present. However, if a singularity is exposed in vacuum, the problem will become very serious.

The calculation in this paper proves that the singularities would appear at the center of a thin loop and the contact point of two spheres according to the Einstein's theory of gravity. The singularities would be exposed in vacuum completely. The space nearby the surfaces of thin circle and double spheres were high curved. Theses are impossible completely. If they were true, we could held a black hole in our hand by bending a fine wire into a circle or griping two spheres together. The ruler would be bended when it was placed in the center region of a thin loop. Light would bend and the effect of gravitational lenses would be seen when it passed through the central region of finger circle. These results are obviously unimaginable and absorbed.

So the singularity in the Einstein's theory of gravity can only be caused by the description method of curved space-time. By the rational analogy, the so-called singular black holes, white holes and wormholes which connect both holes in the current cosmology and astrophysics are something illusive. They can not exist in the real world. The true world excludes infinites. If there are black holes in nature, they can only be the types of the Newtonian black holes without singularity.

In fact, a correct theory of physics can not tolerate the existence of infinites. It is well known that the history of physics is the one to overcome infinites. Modern physics grows up in the process to surmount infinites. Physicists and cosmologists should take cautious and incredulous attitude on the problems of singularity. We should think in deep, whether or not our basic theory of gravity has something wrong when we enjoy its so-called beauty and symmetry.

In order to escape the puzzle of singularity thoroughly, the description of gravity should return to traditional form of dynamics in flat space. Only in this way, the renormalization of gravity can be possible. The unified description of four basic interactions can be possible only based on the frame of flat space-time. Otherwise, based on curved space-time, theses problems can not be solved further. Physicists should understand this situation clearly.

\section{REFERENCES}

[1] X. C. Mei, International Journal of Astronomy and Astrophysics, Vol. 1, 2011, pp. 109-116. doi:10.4236/ijaa.2011.13016

[2] R. E. Schild, et al., The Astronomical Journal, Vol. 132, 2006, p. 420. doi:10.1086/504898

[3] X. Mei and P. Yu, International Journal of Astronomy and Astrophysics, Vol. 2, 2012, pp. 6-18.

[4] R. P. Kerr, Physical Review Letters, Vol. 11, 1963, pp. 237-238. doi:10.1103/PhysRevLett.11.237

[5] E. T. Newman, E. Couch, K. Chinnapared, A. Exton, A. Prakash and R. Tarrence, Journal of Mathematical Physics, Vol. 6, 1965, p. 918. doi:10.1063/1.1704351

[6] S. W. Hawking and G. F. R. Eills, The Large Scale Structure of Space Time, 1972. 